\title{
Preparing Scientists for Science Diplomacy Requires New Science Policy Bridges
}

\author{
Meredith L. Gore, ${ }^{\mathrm{a}, 1}$ Elizabeth S. Nichols, ${ }^{\mathrm{b}}$ and Karen R. Lips ${ }^{\mathrm{c}}$ \\ a Department of Geographical Sciences, University of Maryland College \\ Park, College Park, MD, United States \\ b Department of Ecology, Biosciences Institute, University of São Paulo, \\ São Paulo, Brazil \\ c Department of Biology, University of Maryland College Park, College Park, \\ MD, United States \\ gorem@umd.edu; lizsnichols@gmail.com;klips@umd.edu
}

Received: 19 May 2020; revised: 19 June 2020; accepted: 11 July 2020

\section{Summary}

Differences between the outputs of academic science and those of science policy contribute to a critical science-policy challenge - the inability of academia to sufficiently value either the outputs of the policy process as comparable to academic outputs, or the expertise required to maintain and develop policy. Few colleges and universities in the United States adequately prepare students to become scientists with expertise operating in science-policy spaces. Consequently, most academic scientists lack sufficient training in the policy process, exposure to science diplomacy and capacity to deliver science advice. Science-policy relationships are more than the dichotomised paradox of politicisation of science and the scientisation of politics. Adjustments in how scientists teach, research and engage with policy and policy-makers are necessary to better prepare future generations to address global problems. This article describes currency variances used in these two ecosystems and identifies opportunities to better support science-policy collaborations for more effective research, teaching and service.

1 All three authors provided equal contributions to this article. The views and opinions expressed in this article are those of the authors and do not necessarily reflect the official policy or position of any agency of the US government. Assumptions made within the analysis are not a reflection of the position of any US government entity. 


\section{Keywords}

academia - boundary spanning - science engagement - science policy - science, technology, engineering and mathematics (STEM) - higher education

\section{Introduction}

Global problems related to the environment, human health, technology and computing, national security and international affairs are complex and synergistic. Their effective solutions require collaborations between policy-makers and researchers from multiple disciplines, working across geopolitical boundaries and at multiple levels of governance. Such successful collaborations rely on institutions and a problem-solving mentality that depends on boundaryspanning individuals who share vocabulary, values and practices with academic scientists, diplomats, legislators and policy practitioners. ${ }^{2}$ Boundary-spanning scientists understand and appreciate the values and cultures of policy-makers, develop effective communication skills, learn new vocabularies and are able to function in a policy ecosystem distinct from that where scientists are trained. Global developments create opportunities for foreign diplomacy and science policy, including, inter alia, increased diplomatic engagement, international research coordination and policy coherence ${ }^{3}$ related to the COVID-19 pandemic (e.g., biomedical testing, epidemiology, wildlife disease and trafficking). However, few universities adequately prepare students to operate in these spaces ${ }^{4}$ and, as a result, most academic scientists lack training in the policy process, opportunities to interact with science negotiators and capacity to leverage their scientific expertise to advance either policy or diplomacy. ${ }^{5}$ This limits the academic experience of students, restricts the research impact of scientists, hampers science-policy interactions and science diplomacy, degrades science advice and minimises engagement of universities in domestic and international contexts.

After taking leaves of absence from tenure-track faculty positions to serve at the United States Department of State as Jefferson Science (KRL and MLG) and American Association for the Advancement of Science Technology and Policy (ESN) Fellows, the authors see the importance of increased participation

2 Bednarek et al. 2016, 15.

3 Young, Flink and Dall 2O2O, 1-2.

4 National Academies of Sciences, Engineering, and Medicine 2018, 15-28.

5 Akerlof et al. 2019, 10. 
by scientists in international science advice, science diplomacy and policy development (e.g., scientific contributions of biodiversity scientists to the Intergovernmental Science-Policy Platform on Biodiversity and Ecosystem Services), as well as their own lack of academic preparation for working with policy professionals and diplomats. As university professors in the United States, the authors recognise a gap between content taught in higher education, the process of scientific research and what successfully executed science-diplomacy engagement and science policy involves. Science-policy relationships are more than the dichotomised paradox of politicisation of science and the scientisation of politics. The authors believe that adjustments in how scientists teach, research and engage with policy and policy-makers are necessary to better prepare future generations to address global problems. Here, the authors describe differences in currencies used in these two ecosystems and identify opportunities for academics to better support science-policy collaborations in their research, teaching and service through their United States-based lens.

\section{Two Worlds, Two Currencies}

While scientific research and science policy share a goal of incremental progress towards a better understanding and management of the world, they are developed in distinct ecosystems, with unique institutional cultures in which impact is assessed through completely different metrics. Success in academic science is predicated on the production of individual, discrete scholarly prod$u c t s$, with a relatively linear and causal relationship between input (e.g., financing, expertise, time) and output (e.g., peer-reviewed papers, original books, extramural grants, PhD students graduated). In contrast, success in science policy largely depends on the creation and maintenance of institutional decisions, governance agreements, interpersonal relationships and information flows between science and policy worlds. In this system, the time and effort invested in building coalitions, developing trust and creating a foundation of common understanding are the key products of most policy efforts. This process depends on building and sustaining relationships with diverse stakeholders and consensus building across various levels of government authority, all the while accommodating new ideas, new political and diplomatic developments and changes in funding. This 'constant gardening' is essential to maintain and update existing policies, create new policy and enable legislative uptake of scientific and technical information. Instead of the discrete and quantifiable products of academia, the relationships between policy inputs 
(e.g., financing, expertise, time, relationships) and outputs (i.e., policies, relationships, engagement) are usually non-linear and diffuse. In policy-making, the primary product is the process.

These differences between the outputs of academic science and those of science policy contribute to a critical science-policy challenge - the inability of academia to sufficiently value either the outputs of the policy process as comparable to academic outputs, or the expertise required to maintain and develop policy. Because the academic reward system is not designed to enumerate and value the intangible results of the science-policy process, it seldom rewards scientists who work in policy or diplomacy spheres, even if the scientist's work is 'seen.' The reward system maintains this status quo, even if administrative leaders seek alternatives. Below, the authors describe their convergent observations of systemic changes across all three of the traditional academic areas of teaching, research and service that could more directly produce graduates with skills to effectively span the disciplinary boundaries between science, policy and diplomacy. Their conclusions derive from their diverse approaches and previous work straddling academia and science policy (see Fig. 1 below).

\section{3}

\section{Teach the Process}

Teaching that promotes engagement with real-world issues, problem-solving approaches and interpersonal skills is greatly needed to address global problems but such courses remain underrepresented in science, technology, engineering and mathematics (STEM) curricula. ${ }^{6}$ The same metrics that contribute to a productive academic research career often contribute to learning environments that laud specialisation and technical skills at the cost of skills that are fundamental to the policy-making process. These include how to analyse and articulate policy issues, relationship building, creating trust and learning the values, vocabulary and practices of other fields. The strict focus on disciplinary knowledge derives from the perception that graduates must have extensive technical skills to get a job. Although STEM graduates with strong interpersonal skills, leadership and problem-solving abilities are more competitive on the job market, ${ }^{7}$ these skills are rarely included in sтEм curricula. ${ }^{8}$ The realities of an increasingly competitive and limited academic job market expose

6 National Academies of Sciences, Engineering, and Medicine 2018, 63-9o.

7 Goring et al. 2014, 40.

8 National Academies of Sciences, Engineering, and Medicine 2018, 29-62. 

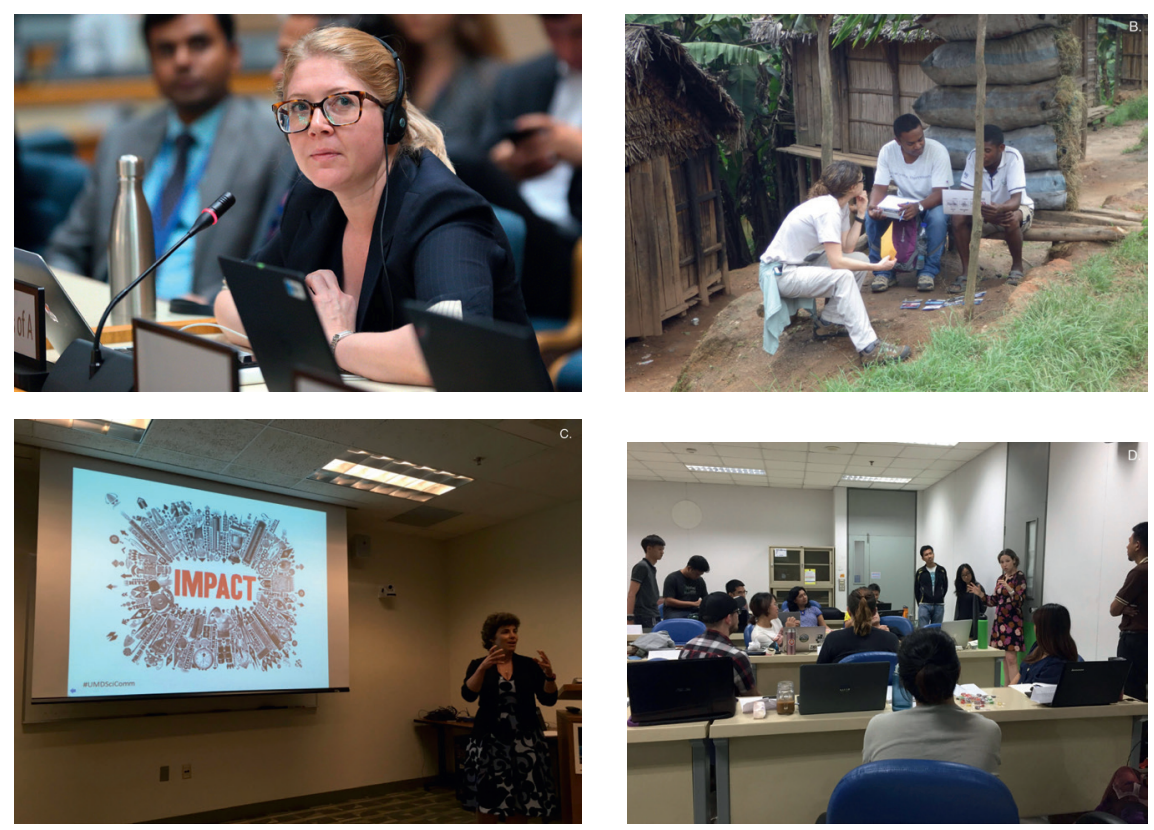

FIGURE 1 Examples of how teaching, research and engagement components of academic science contribute to policy. (A) Liz Nichols interviewing at the Fourth United Nations Environmental Assembly in Nairobi, Kenya (photograph by International Institute for Sustainable Development/Earth Negotiations Bulletin | Mike Muzurakis); (B) Meredith Gore conducting interviews on wildlife trafficking in Madagascar (photograph by Amanda Lewis); (C) Karen Lips teaching a workshop in Scholarly Communication and Public Engagement at the University of Maryland (photograph by Melissa Kenney); (D) Meredith Gore leading a conservation social science workshop for conservation ecologists in Bangkok, Thailand (photograph by Tewa Japf).

graduates to a case of 'double jeopardy,' where universities prepare students for traditional academic careers that only a small fraction will achieve, and simultaneously limit exposure to courses and experiences that would prepare them to work across a range of science-policy or diplomacy spaces.

A rethinking of program curricula at all levels is necessary to produce graduates capable of occupying critical science-policy positions. ${ }^{9,} 10$ Opportunities abound for innovation in enhancing teaching practices and content in the sciences, ${ }^{11}$ and faculty could include policy, diplomatic and legislative compo-

$9 \quad$ Bernstein et al. 2017, 861-862.

10 Hering $2018,555^{8}$.

11 Cvitanovic et al. 2018, 8. 
nents in many aspects of the STEM classroom. For example, an environmental engineering class on assessing urban groundwater contamination risks may include environmental justice modules. Academics could co-teach case studies $^{12}$ with policy practitioners or with faculty from other disciplines (e.g., remote sensing of forest loss in the Congo forest basin and geopolitics of peace parks). Faculty could develop courses in which multidisciplinary problemsolving teams (e.g., Hacking 4 Diplomacy) ${ }^{13}$ address relevant issues as a mechanism to learn how communication, leadership and interpersonal skills are needed to apply technical and disciplinary knowledge. ${ }^{14}, 15$ For example, the authors have used the simulations produced by the National Museum of American Diplomacy on 'Crisis in the Oceans' to demonstrate the multidisciplinary expertise needed in negotiation and conflict resolution. ${ }^{16}$ Graduate and postdoctoral education could include direct training in science policy, transactional communication skills and working with decision-makers ${ }^{17}$ such as exemplified by the Society for Conservation Biology's David H. Smith Conservation Research Fellowship Program. ${ }^{18}$ Universities can reward faculty who develop courses that promote multidisciplinary collaboration across geographic localities and academic disciplines (e.g., machine learning algorithms modelling the spread of chytrid fungus on amphibian population collapse), or who offer training in public engagement, science diplomacy and scholarly communication. Failing to capitalise on these opportunities ignores student needs for diverse professional skills as well as growing demands that higher education show evidence of societal values of college degrees.

Integrate Policy-makers into Research

Policy-relevant, evidence-based science is desperately needed to respond to global problems. While most research products are never cited by either scientists or policy-makers, ${ }^{19}$ with additional training, awareness and engagement across stakeholder groups, a larger proportion of research could have impact

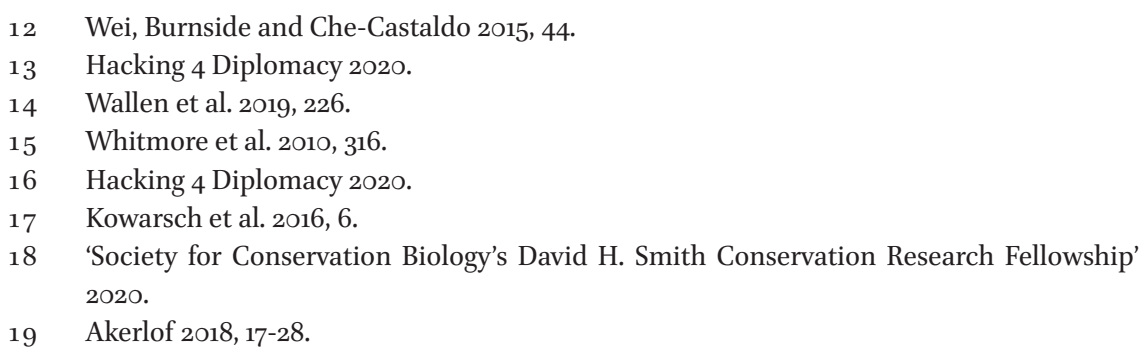


beyond the walls of academia. ${ }^{20,}{ }^{21}$ This requires that STEM scientists recognise where science can and cannot contribute to policy (e.g., dam removal science versus policy funding for the activity), how to distinguish whether scientific expertise or diplomatic relationship building is more likely to achieve outcomes and how to collaborate across disciplines and produce useful information (including, but not limited to, data) for practitioners, legislators and policy-makers. For example, best practices in evidence-based decisionmaking 22 are heavily influenced by the social and behavioural sciences and include using stakeholder mapping, co-developing research questions with stakeholders, using participatory approaches, involving knowledge brokers in implementation and establishing knowledge management systems. ${ }^{23}$ Beyond enriching the research process, these practices would introduce researchers to and help sustain them within innovative programs, collaborations and lines of funding.

New academic incentive structures that encourage, reward and sustain efforts to expand scientific research beyond the ivory tower are needed to establish a science-policy interface that is more responsive to global problems and a diplomatic corps that is increasingly able to interface with scientific evidence. Examples might include revising departmental policy on the promotion and tenure metrics to reflect time spent on public engagement, science advice, scholarly communication, diplomacy labs, participation in collaborations with policy-makers or use of research in policy briefs. Universities might consider hosting policy-makers or legislators on campus as visiting lecturers or offering paid sabbaticals for faculty to work in government service. Ultimately, academic science - particularly within ST EM departments - needs to critically examine the application and use of scholarly activities in the service of society, and the implications of such engagement for institutions, society and individual scientists. Such efforts would reflect the clear desire from policymakers for increased engagement by researchers - for example, the National Science Foundation's requirement that all grants include broader impacts makes clear the link between funding success and public engagement (of

\footnotetext{
$20 \quad$ Whitmore et al. 2010, 315.

21 Chowdhury, Koya and Philipson 2016, 4.

22 Cvitanovic, McDonald and Hodbay 2016, 866.

23 Trapido $2015,1498$.
} 
which science policy and science diplomacy are but two examples). Efforts to further quantify the financial and reputational benefits accrued by universities from public engagement would accelerate changes in the academic reward system, as would quantifying student demand for socially relevant curricula. Communication is at the centre of these dynamics; the capacity to communicate effectively across disciplines and among different stakeholder groups is a necessary tool for diplomacy, and science diplomacy especially. These shifts require an expansion beyond the current STEM-centric model towards a model that recognises the reciprocal interactions between science and society, and rewards the process of change-making. This too, is a kind of diversification of academic departments and is reflected at the institutional level in the creation of new positions that oversee campus efforts in engagement and interdisciplinary programs and centres. Critically, broadening the definition of what counts as scholarship in academia would work towards mitigating the growing distrust between the public and higher education, and help justify the investment in research and discovery by funding agencies.

Getting to the 'world we want' will require institutionalising dynamic and bidirectional exchanges between science and policy to promote a healthier exchange between universities and society (see Fig. 2 below). Recognition of the role of boundary-spanning individuals and organisations is increasing not just scientists identified as novel innovators in knowledge creation for new spaces ${ }^{24}$ - reflecting their critical role in facilitating conversations, connecting centres of excellence and effecting change. ${ }^{25,26}$ The authors posit that recognising the policy process as an academic product is a key science-policy innovation, and that university leadership can mainstream and support this innovation at low financial cost with changes to curricular priorities, measures of research progress and metrics of career advancement. Likely, many valuable lessons can be learned from what land grant universities and historically black colleges and universities experienced in engagement. ${ }^{27}$ The CoviD-19 pandemic has already begun to strain institutional budgets, and generate conversation about the future of academic institutions. As we look towards what elements to maintain, rethink and rebuild, the time has never been more opportune for academia to reflect on more intentional engagement with the world outside the ivory tower.

\footnotetext{
24 Trapido 2015, 1489-149o.

25 Boswell and Smith 2017, 45

26 Bednarek et al. 2016, 14-17.

27 Keeler et al. 2017, 591-592.
} 


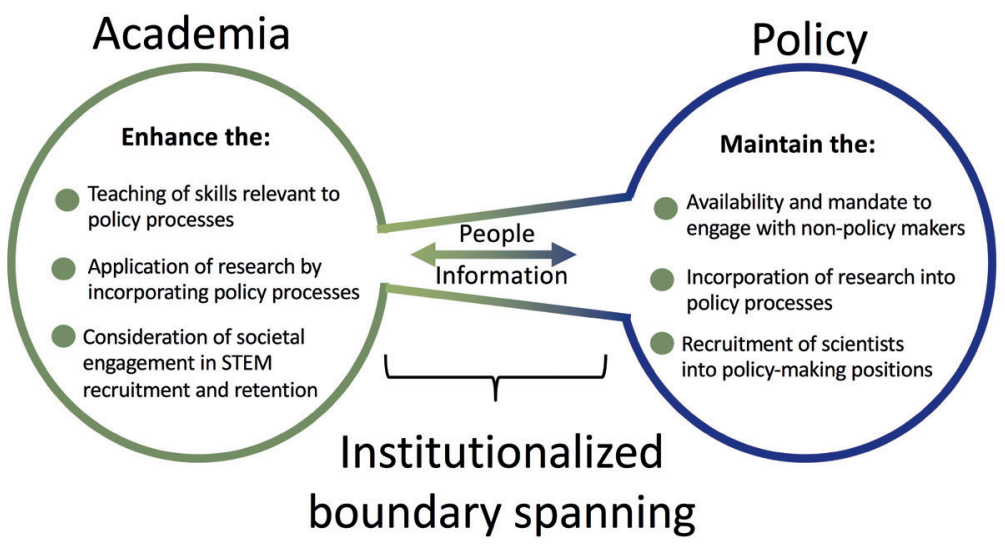

FIGURE 2 Asymmetric recognition of the value of the process and expertise of the policy ecosystem limits academia's contribution to the institutionalisation of boundary spanning, particularly within STEM disciplines. Discrete changes at the laboratory, classroom, departmental and institutional levels in teaching, research and recruitment and retention could increase student exposure to science-policy processes as a form of research application and a potential career avenue, while positively contributing to the institutionalisation of a more stable and sustainable science-policy interface (illustration by the authors).

\section{Bibliography}

Akerlof, Karen. Congress's Use of Science: Considerations for Science Organizations in Promoting the Use of Evidence in Policy (Washington, DC: American Association for the Advancement of Science, 2018).

Akerlof, Karen, Chris Tyler, Sarah Elizabeth Foxen, Erin Heath, Marga Gual Soler, Alessandro Allegra, Emily T. Cloud et al. 'A Collaboratively Derived International Research Agenda on Legislative Science Advice'. Palgrave Communications 5 (1) (2019), 108-121.

Bednarek, Angela, Carina Wyborn, Ryan Meyer, Adam Parris, Peat Leith, Bridie McGreavy and Melanie Ryan. Practice at the Boundaries: Summary of a Workshop of Practitioners Working at the Interfaces Science, Policy and Society for Environmental Outcomes (Pew Charitable Trust, 2016). https://www.pewtrusts.org/ /media/ assets/2016/o7/practiceattheboundariessummaryofaworkshopofpractitioners.pdf.

Bernstein, Michael J., Kiera Reifschneider, Ira Bennett and Jameson Wetmore. 'Science Outside the Lab: Helping Graduate Students in Science and Engineering Understand the Complexities of Science Policy'. Science and Engineering Ethics 23 (3) (2017), 861-882. 
Boswell, Christina and Katherine Smith. 'Rethinking Policy "Impact": Four Models of Research-Policy Relations'. Palgrave Communications 3 (2017), 44-54.

Chowdhury, Gobinda, Kushwanth Koya and Pete Philipson. 'Measuring the Impact of Research: Lessons from the UK's Research Excellence Framework 2014'. PLOS ONE 11 (6) (2016), 1-15.

Cvitanovic, Christopher, Marie F. Löf, Albert v. Nordström and Mark S. Reed. 'Building University-Based Boundary Organizations that Facilitate Impacts on Environmental Policy and Practice'. PLOS ONE 13 (9) (2018), 1-19.

Cvitanovic, Christopher, Jan McDonald and Aysha Hodbay. 'From Science to Action: Principles for Undertaking Environmental Research that Enables Exchange and Evidence-Based Decision-Making'. Journal of Environmental Management 183 (3) (2016), 864-874.

Goring, Simon J., Kathleen C. Weathers, Walter K. Dodds, Patricia A. Soranno, Lynn C. Sweet, Kendra S. Cheruvelil, John S. Kominski et al. 'Improving the Culture of Interdisciplinary Collaboration in Ecology by Expanding Measures of Success'. Frontiers in Ecology and Environment 12 (1) (2014), 39-47.

Hacking 4 Diplomacy. "Learn How to Apply "Lean Startup" Principles to Tackling Global Challenges'. 2020. http://web.stanford.edu/class/msande298/.

Hering, Janet G. 'Implementation Science for the Environment'. Environmental Science and Technology $5_{2}$ (2018), 5555-556o.

Keeler, Bonnie L., Rebecca Chaplin-Kramer, Anne D. Guerry, Prue F.E. Addison, Charles Bettigole, Ingrid C. Burke, Brad Gentry et al. 'Society Is Ready for a New Kind of Science - Is Academia?' Bioscience 67 (7) (2017), 591-592.

Kowarsch, Martin, Jennifer Garad, Pauline Riousset, Dominic Lenzi, Marcel J. Dorsch, Brigitte Knoph, Jan-Albrecht Harrs et al. 'Scientific Assessments to Facilitate Deliberative Policy Learning'. Palgrave Communications 2 (2016), 1-20.

National Academies of Sciences, Engineering, and Medicine. Graduate STEMEducation for the 21st Century (Washington, DC: National Academies Press, 2018).

'Society for Conservation Biology's David H. Smith Conservation Research Fellowship Program'. Society for Conservation Biology, 2020. https://conbio.org/mini-sites/ smith-fellows.

Trapido, Dennis. 'How Novelty in Knowledge Earns Recognition: The Role of Consistent Identities'. Research Policy 44 (8) (2015), 1488-1500.

Wallen, Kenneth E., Karen Filbee-Dexter, Jeremy B. Pittman, Stephen M. Posner, Steven M. Alexander, Chelsie L. Romulo, Drew E. Bennett et al. ‘Integrating Team Science into Interdisciplinary Graduate Education: An Exploration of the SESYNC Graduate Pursuit'. Journal of Environmental Studies and Sciences 9 (2) (2019), 218-233. 
Wei, Cynthia A. William R. Burnside and Judy P. Che-Castaldo. 'Teaching Socio-

Environmental Synthesis with the Case Studies Approach'. Journal of Environmental Studies and Sciences 5 (1) (2015), 42-49.

Whitmore, Ali, Laura Ogden, John Lawton, Pam Sturner, Peter M. Groffman, Laura

Schneider, David Hart et al. 'The Engaged University: Providing a Platform for Research That Transforms Society'. Frontiers in Ecology and the Environment 8 (6) (2010) 314-321.

Young, Mitchell, Tim Flink and Elke Dall. Science Diplomacy in the Making: Case-Based Insights from the $\mathrm{S}_{4} \mathrm{D}_{4} \mathrm{C}$ Project (Vienna: Centre for Social Innovation, 202O).

\section{Meredith L. Gore}

earned her $\mathrm{PhD}$ in natural resources policy and management from Cornell University. She is a conservation social scientist using interdisciplinary approaches to understand human-environment relationships. Her research is global in scope and focuses on environmental risks and transnational environmental crime (e.g., wildlife trafficking, illegal logging, illegal fishing). Her areas of interest are community-based management, environmental risk perception, science diplomacy and interdisciplinary conservation science.

\section{Elizabeth S. Nichols}

earned her PhD in ecology from Columbia University. As a conservation ecologist, her research program explores tropical biodiversity responses to conservation and management practices and policies. As policy-maker, she serves in the US Department of State as a Foreign Affairs Officer where she manages the science-policy basis of the US relationship with the Global Environment Facility, and represents the United States in international environmental negotiations.

\section{Karen R. Lips}

earned her PhD in biology from the University of Miami. She is an ecologist who studies how global change affects biodiversity in the United States and in Latin America. A primary focus of her research is determining the ecological and environmental factors that influence amphibian responses to disease, and how that information might be used in conservation and recovery plans. She is also interested in increasing engagement on environmental issues, promoting scientific leadership and fostering international scientific collaborations. 